\title{
Zahnerosionen im Zusammenhang mit gastroösophagealem Reflux: Ursache, Prävention und restaurative Therapie
}

\author{
Wegehaupt, Florian J ; Attin, Thomas
}

\begin{abstract}
Tooth Erosions Associated with Gastroesophageal Reflux: Cause, Prevention and Restorative Therapy Abstract. Dental erosions are caused by the recurring contact of solutions which are unsaturated in tooth minerals,ith hard tooth substances. This initially leads to softening and later to an irreversible loss of hard tooth substance. Erosion is observed particularly with excessive consumption of acidic foods (e.g. soft drinks or citrus fruits) but also in connection with gastrointestinal (gastroesophageal reflux disease) or psychosomatic diseases (anorexia nervosa or bulimia nervosa). The aim of this article is to define dental erosions, their causes, prevalence and consequences as well as possible preventive measures. Based on a clinical example of a patient with reflux-related erosions, a therapy option with direct composite tooth build-up is presented
\end{abstract}

DOI: https://doi.org/10.1024/1661-8157/a003184

Other titles: Tooth Erosions Associated with Gastroesophageal Reflux: Cause, Prevention and Restorative Therapy

Posted at the Zurich Open Repository and Archive, University of Zurich ZORA URL: https://doi.org/10.5167/uzh-181480

Journal Article

Accepted Version

Originally published at:

Wegehaupt, Florian J; Attin, Thomas (2019). Zahnerosionen im Zusammenhang mit gastroösophagealem Reflux: Ursache, Prävention und restaurative Therapie. Praxis, 108(5):307-313.

DOI: https://doi.org/10.1024/1661-8157/a003184 
Zahnerosionen im Zusammenhang mit gastroösophagealem Reflux:

Ursache, Prävention und Restaurative Therapie.

PD Dr. med. dent. Florian J. Wegehaupt

Prof. Dr. med. dent Thomas Attin

Universität Zürich

Zentrum für Zahnmedizin

Klinik für Präventivzahnmedizin, Parodontologie und Kariologie

Plattenstrasse 11

8032 Zürich

Schweiz 


\section{Zusammenfassungen:}

Dentale Erosionen entstehen durch den wiederkehrenden Kontakt von Lösungen, die an Zahnmineralien ungesättigt sind, mit den Zahnhartsubstanzen. Dieses führt initial zu einer Erweichung und später zu einem irreversiblen Verlust an Zahnhartsubstanzen. Erosionen werden insbesondere beim übermässigen Konsum saurer Lebensmittel (z.B. Softdrinks oder Zitrusfrüchte) aber auch im Zusammenhang mit gastro-intestinalen Erkrankungen (gastroösophageale Reflux-Erkrankung) oder psychosomatischen Erkrankung (Anorexia nervosa oder Bulimia nervosa) beobachtet.

Ziel des vorliegenden Artikel soll es sein, dentale Erosionen zu definieren und ihre Ursachen, Prävalenz und Folgen sowie mögliche präventive Massnahmen aufzuzeigen. Anhand eines klinischen Beispiels eines Patienten mit Reflux-bedingten Erosionen wird eine Therapiemöglichkeit mit direktem Kompositaufbauten der Zähne dargestellt.

\section{Schlüsselwörter:}

dentale Erosionen, gastroösophagealer Reflux, Prävention, restaurative Therapie 


\section{Haupttext:}

\section{Ü1 Definition und Folgen dentaler Erosionen:}

Klassischer Weise werde dentale Erosionen als chemische, oberflächliche und irreversible Zahnhartsubstanzverluste definiert, die durch Säuren verursacht werden. Der Prozess des Zahnhartsubstanzverlustes kann den Zahnschmelz, das Dentin und das Wurzelzement betreffen und findet ohne Beteiligung von Mikroorganismen statt [1]. Neuerdings spricht man nicht mehr zwangsläufig davon, dass diese Art von Zahnhartsubstanzverlust durch Säuren verursacht wird, sondern davon, dass der Kontakt mit Lösungen/Produkten, die an Zahnmineralien untersättigt sind, zu Erosionen führen [2]. Diese Definition trägt dem Umstand Rechnung, dass destilliertes Wasser, welches ja keine Mineralien enthält, dafür aber einen eher neutralen pH-Wert aufweist, Zahnhartsubstanzen ebenfalls demineralisieren kann, wohingegen Joghurt mit einem sauren pH-Wert, aufgrund seiner Übersättigung mit Calcium und Phosphat, keine Erosionen verursacht [3]. Unter physiologischen Mundhöhlenbedingungen ist Schmelz bis zu einem pH-Wert von 5.5 stabil [4]. Erst darunter findet eine Demineralisation statt. Die Säureeinwirkung bzw. der Kontakt mit den an Zahnmineralien ungesättigten Lösungen führt zu einer Demineralisierung, die sich zu Beginn in einer Erweichung der Zahnhartsubstanz zeigt [5]. Bei längeren und/ oder häufigeren Säurekontakten kommt es zu einem irreversiblen Volumenverlust und zu klinisch sichtbaren Defekten [6, 7]. Bei einer beginnenden dentalen Erosionen, aber auch nach einem irreversiblen Volumenverlust, findet sich eine erweichte Zahnoberfläche, welche empfindlich gegenüber mechanischen Angriffen wie zum Beispiel dem Zähneputzen ist [8, 9]. Dieser Umstand führt dazu, dass klinisch häufig eine „Mischung“ aus erosivem und abrasivem (durch ein weiteres Medium mechanisch verursachter Zahnhartsubstanzverlust) Schaden zu finden ist.

Die ersten klinischen Zeichen von Erosionen sind eine glatte seidenglänzende bis matte Zahnoberfläche. Auf Glattflächen der Zähne flachen die Konvexitäten ab oder es werden 
sogar Konkavitäten sichtbar. Initiale Läsionen zeigen sich koronal der Schmelz-ZementGrenze mit einer intakten Schmelzleiste am Gingivarand. In fortgeschrittenen Stadien zeigt sich eine veränderte Zahnmorphologie, wobei sich Höckerspitzen und Inzisalkanten abrunden und Zahn-Füllungen höher zu stehen kommen als die benachbarte Zahnsubstanz [10]. Ausgedehnter Schmelzverlust kann zur Dentinfreilegung und in extremen Fällen bis zur Pulpaexposition führen [11]. Die freigelegten Zahnoberflächen werden empfindlich auf Warm und Kalt sowie auf Berührung [12]. Fortgeschrittene Defekte zeichnen sich auch durch einen Verlust an vertikaler Höhe der Zähne aus [6]. Innerhalb von 6 Monaten konnte für eine Patientengruppe, welche unter Erosionen litt, ein Zahnhartsubstanzverlust von durchschnittlich $36.5 \mu \mathrm{m}$ beobachtet werden, wohingegen der Zahnhartsubstanzverlust in der „gesunden“ Kontrollgruppe nur 3.7 mm betrug [13].

\section{Ü1 Ursache dentaler Erosionen:}

Erosionen werden aufgrund des Ursprunges der Erosions-verursachenden Säure bzw. der an Zahnmineralien ungesättigten Lösungen, die entweder extrinsischen (von ausserhalb des Körpers) oder intrinsischen (aus dem Körper) Ursprungs sein kann, unterschieden.

Extrinsische Säuren können durch den Konsum von Nahrungsmitteln (Zitrusfrüchte, Softdrinks, Wein, Salatdressing, etc.) [14] und Medikamenten (Acetylsalicylsäure, Eisentabletten, Vitamin-C-Supplemente, etc.) [15], aber auch durch beruflich bedingte Säureexposition (Batteriefabrik, Weintester, Berufsschwimmer, etc.) [16, 17], Zahnerosionen verursachen.

Als einzige Quelle für eine intrinsische Säure kommt die Magenflüssigkeit, welcher hauptsächlich aus Salzsäure $(\mathrm{HCl})$ besteht [18], in Frage. Verschiedene Formen von Essstörungen (Anorexia nervosa, Bulimia nervosa) [19], gastro-intestinale Erkrankungen wie die gastroösophageale Reflux-Erkrankung (engl. GERD: gastroesophageal reflux 
disease) [20] und Alkoholabusus [21] sind bekannte Ursachen dafür, dass saurer Mageninhalt bzw. Magensaft über den Ösophagus in die Mundhöhle gelangt. Bei der gastroösophagealen Reflux-Erkrankung treten, vor allem nachts, Reflux-Perioden auf, bei denen der pH-Wert im Oesophagus für bis zu 10 Minuten auf pH 1 abfallen kann [22]. Beim gastroösophagealen Reflux, welcher auch physiologisch vorkommen kann, fällt der pH-Wert für mindestens 30 Sekunden unter pH 4 ab [23]. Eine Studie aus dem Jahr 1996 konnte zeigen, dass bei Patienten mit einer gastroösophagealen Reflux-Erkrankung der orale $\mathrm{pH}$-Wert bei einer 24 Stunden $\mathrm{pH}$-Wert Messung während $0.3 \%$ der Zeit unter $\mathrm{pH}$ 5.5 (unter physiologischen Bedingungen kritischer pH-Wert für Schmelz [4]) und während 4.4\% der Zeit unter pH 6 abfällt. Dieses entspricht somit einer Erosionsdauer von 4.3 bis 60 min pro Tag [24].

\section{Ü1 Prävalenz:}

In einer „gesunden“ Gesamtbevölkerung wurden für drei- bis sechs-jährige Kinder im Jahr 2004/05 Prävalenzen von dentalen Erosionen zwischen 22\% und 38\% beobachtet [25]. Zehn Jahre später (2005/06) betrug die Prävalenz bei den drei- bis sechs-jährigen Kindern $14 \%$ bis $71 \%$ [26]. Für Erwachsene im Alter von 26-30 Jahren wird eine Prävalenz von rund $30 \%$ und für $46-50$-jährige eine solche von $43 \%$ angegeben [27].

Für Kinder, die unter einer gastroösophagealen Reflux-Erkrankung leiden, wurde eine Prävalenz dentaler Erosionen von 98\% beobachtet, wohingegen in der „gesunden“ Kontrollgruppe nur eine Prävalenz von 19\% festgestellt wurde [28]. Auch für erwachsene Patienten, die unter einer gastroösophagealen Reflux-Erkrankung leiden, wird eine deutlich erhöhte Prävalenz (75\%) an dentalen Erosionen im Vergleich zur gesunden Kontrollgruppe (17\%) angegeben [29].

Ebenso wird für Patienten, die unter Essstörungen (Anorexia nervosa, Bulimia nervosa) leiden, eine deutlich erhöhte Prävalenz (45\% [30] - 98\% [31]) an dentalen Erosionen 
beobachtet [32]. Unabhängig von der Art der Essstörung wurde für diese Patienten ein 8.5-fach höheres Risiko für dentale Erosionen ermittelt [33].

\section{Ü1 Prävention:}

Präventive Massnahmen zum Schutz vor erosivem Zahnhartsubstanzverlust bzw. der mit der Erosion einhergehenden Erweichung der Zahnhartsubstanzen sollten primär darauf Abzielen, den Kontakt der Zähne mit den verursachenden Agenzien (z.B. saure Getränke, Magensaft usw.) zu reduzieren bzw. einzuschränken. Diese bedeutet im Fall von externen Erosionen eine entsprechende Aufklärung der Patienten mit entsprechender Ernährungslenkung. Im Falle von Erosionen im Zusammenhang mit gastro-intestinalen Erkrankungen oder psychosomatischen Erkrankung wie z. B. Anorexia nervosa und Bulimia nervosa ist eine Überweisung des Patienten an Kollegen der entsprechenden Fachdisziplinen (Gastroenterologie bzw. Psychatrie) indiziert um die zugrundeliegende Grunderkrankung zu therapieren.

Neben diesen primär-präventiven Massnahmen sind verschiedene andere Ansätze zur Reduktion der klinischen Symptome (Zahnhartsubstanzverlust) untersucht und beschrieben worden. Diese Massnahmen zielen darauf ab die (Säure-) Löslichkeit der entsprechenden Zahnhartsubstanzen zu reduzieren bzw. die erweichten Zahnhartsubstanzen wieder zu erhärten. Zu dieser Art von präventiven Massnahmen zählt die topische Anwendung verschiedener Fluoridverbindungen [34] (Amin- oder Natriumfluorid), Metallverbindungen [35, 36] (Zinnchlorid oder Titantetrafluorid) oder von Calcium/Phosphat-Verbindungen [37] (Casein Phosphopeptid - amorphes Calciumphosphat) in Form von Zahnpaste, Mundspülungen oder Gelees. Der Erfolg dieser Art von präventiven Massnahmen ist stark von der Compliance der Patienten (regelmässiger Einsatz der entsprechenden Produkte) abhängig. Als Patientencompliance unabhängiger Ansatz wird daher die Versieglung der Zahnhartsubstanzen durch den 
Zahnarzt empfohlen. Dieser Ansatz zielt darauf ab, eine mechanische „Barriere“ auf den Zähnen zu etablieren, welche einen Kontakt der Erosions-verursachenden Lösungen mit den Zahnhartsubstanzen verhindern soll. Hierzu können verschiedene Arten von dentalen Adhäsiven oder Oberflächenversieglern zur Anwendung kommen, die durch den Zahnarzt in der Praxis appliziert werden [38].

\section{Ü1 Restaurative Versorgung eines Reflux-geschädigten Gebisses:}

Häufig führen langjährige erosive Einflüsse, wie beim Reflux, zu starken Zahnhartsubstanzverlusten aller Zähne einer Mundhöhle und einem Absenken der Bisshöhe, der sog. okklusalen vertikalen Dimension, so dass oftmals restaurative Massahmen zur Rekonstruktion des gesamten Gebisses erforderlich werden. Je nach Ausmaß des Zahnhartsubstanzverlustes stehen verschiedene Therapieoptionen zur Auswahl [39]. Diese reichen von einer Abdeckung der freigelegten Areale mit einem Versiegelungsmaterial oder fließfähigem Komposit über restaurative Maßnahmen mit direkten Kompositversorgungen bis hin zu indirekten Rekonstruktionen aus Keramik oder neuartigen Hochleistungs-Kompositen, die zunächst durch einen Zahntechniker oder durch CAD-Verfahren hergestellt und dann vom Zahnarzt eingegliedert werden [40-44]. Die Muskulatur der Patienten gewöhnt sich schnell an die neue Bisshöhe, so dass diese von den Patienten i.d.R. sehr gut akzeptiert wird, ohne dass es zu Problemen am Kiefergelenk kommt [45]

Bei indirekten Restaurationen muss beachtet werden, dass i. d. R. zusätzlich eine Präparation am Zahn zu erfolgen hat, um die Aufnahme des Werkstücks mit ausreichend stabiler Schichtstärke zu ermöglichen. Vorteil indirekter Verfahren ist es, dass die verwendeten (Keramik-)Materialien bei Einhaltung der geforderten Schichtstärke Kompositen in Fragen der Stabilität, des Abnutzungsverhaltens sowie der Farbstabilität meist überlegen sind. Direkte Kompositversorgungen aus, mit denen ausschließlich die 
fehlenden Strukturen eines Zahnes wieder aufgebaut wird, erfordern hingegen meist keine zusätzlichen Präparationen am Zahn. Die modernen hochgefüllten Kompositmaterialien weisen ähnlich gut physikalische Eigenschaften wie Keramiken bzw. die natürliche Zahnhartsubstanz auf [46-50].

Zudem konnte in verschiedenen klinischen Studien (mit durchschnittlich bis 5,5 Jahren Follow-up) und durch unveröffentlichte Daten (nach 11 Jahren Tragedauer) gezeigt werden, dass komplexe Zahnrekonstruktionen auch mit direkte Kompositversorgungen $[41,51-54]$.

Die nachfolgende Falldarstellung stellt die Versorgung eines durch Reflux geschädigten Gebisses mit direkten Kompositversorgungen vor, wie diese häufig in unserer Klinik zur Anwendung kommt. Es sollte aber nicht unerwähnt bleiben, dass bei solch komplexen Situationen die Versorgung mit indirekt im Labor hergestellten Werkstücken einen zwar teureren, aber ebenfalls sehr soliden Therapieansatz dargestellt hätte, der ggf. die ästhetische Situation besser gelöst hätte.

\section{Ü1 Falldarstellung}

Der 23-jährige, geistig leicht retardierte Patient, war von seinem Hauszahnarzt zur Versorgung seines Erosionsgebisses an unsere Klinik im Jahr 2016 überwiesen worden (Abb. 1-4). Aufgrund der finanziellen Situation des Patienten sollte eine kostengünstige Versorgung vorgenommen werden. Aus der Anamnese ergab sich, dass der Patient unter einem Reflux litt, so dass er begleitend eine Therapie bei einem Gastroenterologen begann. Gleichzeitig wurde dem Patienten die Anwendung einer zinnhaltigen Fluoridspüllösung empfohlen (Elmex Erosionsschutz, Gaba/Colgate-Palmolive, Therwil, Schweiz).

Nach Versorgung kleinerer kariöser Läsionen und dem Austausch defekter 
Restaurationen wurden zunächst die Seitenzähne mit Komposit (Filtek XTE, 3M Espe, St. Paul, USA) unter Zuhilfenahme eines Adhäsivs OptiBond FL (Kerr, Orange, USA) aufgebaut (Abb. 5 und 6). Zum Aufbau der Zähne wurde eine Technik verwendet, die durch Mitarbeiter unserer Klinik im Jahre 2006 entwickelt und anschliessend verfeinert wurde. Diese Technik erlaubt es, die Zahnmorphologien mit Kompositmaterialien direkt im Munde des Patienten so wiederherzustellen, dass eine approximale Passage mit Zahnseide zur Reinigung der Zwischenräume weiterhin möglich ist.

In nachfolgenden Behandlungssitzungen wurden die Frontzähne des Unter- und Oberkiefers ebenfalls mit Kompositmaterialien direkt im Munde des Patienten restauriert (Abb. 7-9). Die Abbildung 10 zeigt die okklusale Ansicht der restaurierten Zähne im Oberund Unterkiefer.

Zum Abschluss wurde für den Patienten durch den Zahntechniker eine weiche Tiefziehschiene für die Nachtzeit erstellt, um einen Schutz der Restaurationen vor nächtlichem Knirschen sicherzustellen. In der 1-Jahreskontrolle zeigten sich keinerlei Schäden oder Abnutzungen an den neuen Restaurationen.

\section{Key messages:}

- Dentale Erosionen finden sich sehr häufig (Prävalenz bis zu 98\%) im Zusammenhang mit einer gastroösophagealen Reflux-Erkrankung.

- Die ersten Zeichen einer dentalen Erosion sind der Verlust der Mikrostrukturen der Zähne, die Glattflächen verlieren ihre Konvexitäten und es werden sogar Konkavitäten sichtbar. In fortgeschrittenen Stadien zeigen sich Abrundungen der Höckerspitzen und Inzisalkanten. Bestehende Zahn-Füllungen ragen teilweise über die benachbarte Zahnsubstanz heraus. Der Schmelzverlust kann zur Dentinfreilegung mit Empfindlichkeiten auf Temperaturänderungen sowie auf Berührung einhergehen. 
Fortgeschrittene Erosionsdefekte zeichnen sich auch durch einen Verlust an vertikaler Höhe des gesamten gebisses aus.

- Patienten mit einer gastroösophagealen Reflux-Erkrankung sollten frühzeitig zur Abklärung und ggf. Einleitung einer präventiven und/oder restaurativen Therapie an einen Zahnarzt überwiesen werden.

\section{Hinweis:}

Die hier vorliegende Publikation orientiert sich an früheren Publikationen der Autoren zur selben Thematik und ist in manchen Teilen mit diesen Publikationen identisch $[55,56]$.

\section{Korrespondenzadresse:}

PD Dr. med. dent Florian Wegehaupt

Universität Zürich

Zentrum für Zahnmedizin

Klinik für Präventivzahnmedizin, Parodontologie und Kariologie

Plattenstrasse 11, 8032 Zürich, Schweiz

Tel: +414463432 84, Fax: +41446344308

E-Mail: Florian.Wegehaupt@zzm.uzh.ch

\section{Bibliografie:}

1. Zipkin I, McClure FJ. Salivary citrate and dental erosion; procedure for determining citric acid in saliva; dental erosion and citric acid in saliva. J Dent Res. 1949;28:613-626.

2. Larsen MJ. Chemical events during tooth dissolution. J Dent Res. 1990;69 Spec No:575-80; discussion 634 . 
3. Barbour ME, Lussi A. Erosion in relation to nutrition and the environment. Monogr Oral Sci. 2014;25:143-154.

4. Meurman JH, ten Cate JM. Pathogenesis and modifying factors of dental erosion. Eur $\mathrm{J}$ Oral Sci. 1996;104:199-206.

5. Attin T, Koidl U, Buchalla W, Schaller HG, Kielbassa AM, Hellwig E. Correlation of microhardness and wear in differently eroded bovine dental enamel. Arch Oral Biol. $1997 ; 42: 243-250$.

6. Ganss C. Is erosive tooth wear an oral disease? Monogr Oral Sci. 2014;25:16-21.

7. Ganss C, Lussi A, Schlueter N. The histological features and physical properties of eroded dental hard tissues. Monogr Oral Sci. 2014;25:99-107.

8. Attin T, Zirkel C, Hellwig E. Brushing abrasion of eroded dentin after application of sodium fluoride solutions. Caries Res. 1998;32:344-350.

9. Attin T, Buchalla W, Gollner M, Hellwig E. Use of variable remineralization periods to improve the abrasion resistance of previously eroded enamel. Caries Res. 2000;34:48-52.

10. Wang X, Lussi A. Assessment and management of dental erosion. Dent Clin North Am. 2010;54:565-578.

11. Sivasithamparam K, Harbrow D, Vinczer E, Young WG. Endodontic sequelae of dental erosion. Aust Dent J. 2003;48:97-101.

12. Bartlett DW. The role of erosion in tooth wear: aetiology, prevention and management. Int Dent J. 2005;55:277-284.

13. Bartlett DW, Blunt L, Smith BG. Measurement of tooth wear in patients with palatal erosion. Br Dent J. 1997;182:179-184.

14. Kanzow P, Wegehaupt FJ, Attin T, Wiegand A. Etiology and pathogenesis of 
dental erosion. Quintessence Int. 2016;47:275-278.

15. Hellwig E, Lussi A. Oral hygiene products, medications and drugs - hidden aetiological factors for dental erosion. Monogr Oral Sci. 2014;25:155-162.

16. Arowojolu MO. Erosion of tooth enamel surfaces among battery chargers and automobile mechanics in Ibadan: a comparative study. Afr J Med Med Sci. 2001;30:5-8.

17. Wiegand A, Attin T. Occupational dental erosion from exposure to acids: a review. Occup Med (Lond). 2007;57:169-176.

18. Hunt JN. The composition of gastric juice. J Physiol. 1951;113:419-424.

19. Roberts MW, Tylenda CA. Dental aspects of anorexia and bulimia nervosa. Pediatrician. 1989;16:178-184.

20. Bartlett DW, Evans DF, Smith BG. The relationship between gastro-oesophageal reflux disease and dental erosion. J Oral Rehabil. 1996;23:289-297.

21. Schlueter N, Tveit AB. Prevalence of erosive tooth wear in risk groups. Monogr Oral Sci. 2014;25:74-98.

22. Orr WC. Sleep and gastroesophageal reflux: what are the risks. Am J Med. 2003;115 Suppl 3A:109S-113S.

23. Orr WC. Therapeutic options in the treatment of nighttime gastroesophageal reflux. Digestion. 2005;72:229-238.

24. Bartlett DW, Evans DF, Anggiansah A, Smith BG. A study of the association between gastro-oesophageal reflux and palatal dental erosion. Br Dent J. 1996;181:125131.

25. Wiegand A, Muller J, Werner C, Attin T. Prevalence of erosive tooth wear and associated risk factors in 2-7-year-old German kindergarten children. Oral Dis. 
2006;12:117-124.

26. Tschammler C, Müller-Pflanz C, Attin T, Müller J, Wiegand A. Prevalence and risk factors of erosive tooth wear in 3-6 year old German kindergarten children-A comparison between 2004/05 and 2014/15. J Dent. 2016;52:45-49.

27. Lussi A, Schaffner M, Hotz P, Suter P. Dental erosion in a population of Swiss adults. Community Dent Oral Epidemiol. 1991;19:286-290.

28. Farahmand F, Sabbaghian M, Ghodousi S, Seddighoraee N, Abbasi M. Gastroesophageal reflux disease and tooth erosion: a cross-sectional observational study. Gut Liver. 2013;7:278-281.

29. Tantbirojn D, Pintado MR, Versluis A, Dunn C, Delong R. Quantitative analysis of tooth surface loss associated with gastroesophageal reflux disease: a longitudinal clinical study. J Am Dent Assoc. 2012;143:278-285.

30. Hermont AP, Pordeus IA, Paiva SM, Abreu MH, Auad SM. Eating disorder risk behavior and dental implications among adolescents. Int J Eat Disord. 2013;46:677-683.

31. Ohrn R, Enzell K, Angmar-Månsson B. Oral status of 81 subjects with eating disorders. Eur J Oral Sci. 1999;107:157-163.

32. Milosevic A, Slade PD. The orodental status of anorexics and bulimics. Br Dent J. 1989;167:66-70.

33. Johansson AK, Norring C, Unell L, Johansson A. Eating disorders and oral health: a matched case-control study. Eur J Oral Sci. 2012;120:61-68.

34. Wegehaupt FJ, Sener B, Attin T, Schmidlin PR. Anti-erosive potential of amine fluoride, cerium chloride and laser irradiation application on dentine. Arch Oral Biol. 2011;56:1541-1547. 
35. Schlueter N, Klimek J, Ganss C. Effect of stannous and fluoride concentration in a mouth rinse on erosive tissue loss in enamel in vitro. Arch Oral Biol. 2009;54:432-436.

36. Wiegand A, Magalhaes AC, Attin T. Is titanium tetrafluoride (TiF4) effective to prevent carious and erosive lesions? A review of the literature. Oral Health Prev Dent. 2010;8:159-164.

37. Ranjitkar S, Rodriguez JM, Kaidonis JA, Richards LC, Townsend GC, Bartlett DW. The effect of casein phosphopeptide-amorphous calcium phosphate on erosive enamel and dentine wear by toothbrush abrasion. J Dent. 2009;37:250-254.

38. Wegehaupt FJ, Kanzow P, Wiegand A, Attin T. Eignung von Ober ächenversieglern und Dentinadhäsiven zum Schutz vor erosivem Zahnhartsubstanzverlust. Quintessenz. 2015;66:1051-1054.

39. Loomans B, Opdam N, Attin T et al. Severe Tooth Wear: European Consensus Statement on Management Guidelines. J Adhes Dent. 2017;19:111-119.

40. Wegehaupt FJ, Tauböck TT, Sener B, Attin T. Long-term protective effect of surface sealants against erosive wear by intrinsic and extrinsic acids. J Dent. 2012;40:416-422.

41. Hamburger JT, Opdam NJ, Bronkhorst EM, Kreulen CM, Roeters JJ, Huysmans MC. Clinical performance of direct composite restorations for treatment of severe tooth wear. J Adhes Dent. 2011;13:585-593.

42. Grütter L, Vailati F. Full-mouth adhesive rehabilitation in case of severe dental erosion, a minimally invasive approach following the 3-step technique. Eur J Esthet Dent. 2013;8:358-375.

43. Zahn T, Zahn B, Gerhardt-Szep S, Lauer HCH. Rekonstruktion generalisierter Erosionsschäden durch vollkeramische Restaurationen - ein Fallbericht mit 
Langzeitergebnissen nach 6 Jahren. Dtsch Zahnärztl Z. 2014;69:698-706.

44. Edelhoff D, Beuer F, Schweiger J, Brix O, Stimmelmayr M, Guth JF. CAD/CAMgenerated high-density polymer restorations for the pretreatment of complex cases: a case report. Quintessence Int. 2012;43:457-467.

45. Abduo J, Lyons K. Clinical considerations for increasing occlusal vertical dimension: a review. Aust Dent J. 2012;57:2-10.

46. Palaniappan S, Bharadwaj D, Mattar DL, Peumans M, Van Meerbeek B, Lambrechts $P$. Three-year randomized clinical trial to evaluate the clinical performance and wear of a nanocomposite versus a hybrid composite. Dent Mater. 2009;25:1302-1314.

47. Palaniappan S, Bharadwaj D, Mattar DL, Peumans M, Van Meerbeek B, Lambrechts P. Nanofilled and microhybrid composite restorations: Five-year clinical wear performances. Dent Mater. 2011;27:692-700.

48. Palaniappan S, Elsen L, Lijnen I, Peumans M, Van Meerbeek B, Lambrechts P. Nanohybrid and microfilled hybrid versus conventional hybrid composite restorations: 5year clinical wear performance. Clin Oral Investig. 2012;16:181-190.

49. Mörmann WH, Stawarczyk B, Ender A, Sener B, Attin T, Mehl A. Wear characteristics of current aesthetic dental restorative CAD/CAM materials: two-body wear, gloss retention, roughness and Martens hardness. J Mech Behav Biomed Mater. 2013;20:113-125.

50. Krämer N, Küssner P, Motmaen I, Köhl M, Wöstmann B, Frankenberger R. Marginal quality and wear of extended posterior resin composite restorations: Eight-year results in vivo. J Mech Behav Biomed Mater. 2015;50:13-22.

51. Attin T, Filli T, Imfeld C, Schmidlin PR. Composite vertical bite reconstructions in eroded dentitions after 5.5 years: a case series. J Oral Rehabil. 2012;39:73-79. 
52. Schmidlin PR, Filli T, Imfeld C, Tepper S, Attin T. Three-year evaluation of posterior vertical bite reconstruction using direct resin composite--a case series. Oper Dent. 2009;34:102-108.

53. Ramseyer ST, Helbling C, Lussi A. Posterior Vertical Bite Reconstructions of Erosively Worn Dentitions and the "Stamp Technique" - A Case Series with a Mean Observation Time of 40 Months. J Adhes Dent. 2015;17:283-289.

54. Loomans BAC, Kreulen CM, Huijs-Visser HECE et al. Clinical performance of full rehabilitations with direct composite in severe tooth wear patients: 3.5 Years results. $J$ Dent. 2018;70:97-103.

55. Attin T, Bosch G, Wegehaupt FJ, Mehl A, Wiegand A, Blunck U. Rekonstruktion erosiver Zahnhartsubstanzdefekte mit Komposit. Quintessenz. 2015;66:131-143.

56. Attin T, Tauböck T. Direkte adhäsive Kompositrestaurationen zur Rekonstruktion erosiver Zahnhartsubstanzdefekte. Swiss Dent J. 2017;127:131-143. 


\section{Abbildungen/Tabellen:}

Abb. 1:

Aufsicht der Oberkiefer- (links) und Unterkieferzähne (rechts) des

Patienten mit multiplen erosiven Zahnhartsubstanzdefekten.

Abb. 2:

Der Zahnhartsubstanzverlust an den palatinalen Flächen der Oberkieferfrontzähne ist bereits soweit fortgeschritten, dass nur noch dünne Dentinbrücken über der Pulpa vorhanden sind.

Abb. 3 und 4: $\quad$ Frontalsicht der Unterkiefer und Oberkieferfrontzähne.

Abb. 5:

Die Nahaufnahme der Oberkieferseitenzähne zeigt deutlich den Schmelzverlust auf den Kauflächen und die freigelegten, zum Teil typischen muldenförmigen Erosionsdefekte im Dentin.

Abb. 6:

Situation der Zähne von Abbildung 5 nach Rekonstruktion mit Komposit.

Abb. 7:

Mit Komposit aufgebaute Unterkieferfrontzähne.

Abb. 8:

Zwischenschritt beim Aufbau der Oberkieferfrontzähne mit Komposit unter Trockenlegung der Zähne mit blauem KofferdamGummi.

Abb. 9: Mit Kompost aufgebaute Oberkieferfrontzähne von Abbildung 8 nach Fertigstellung und Politur.

Abb. 10:

Aufsicht der Oberkiefer- (links) und Unterkieferzähne (rechts) des Patienten nach Rekonstruktion der Zähne mit Kompositrestaurationen. 


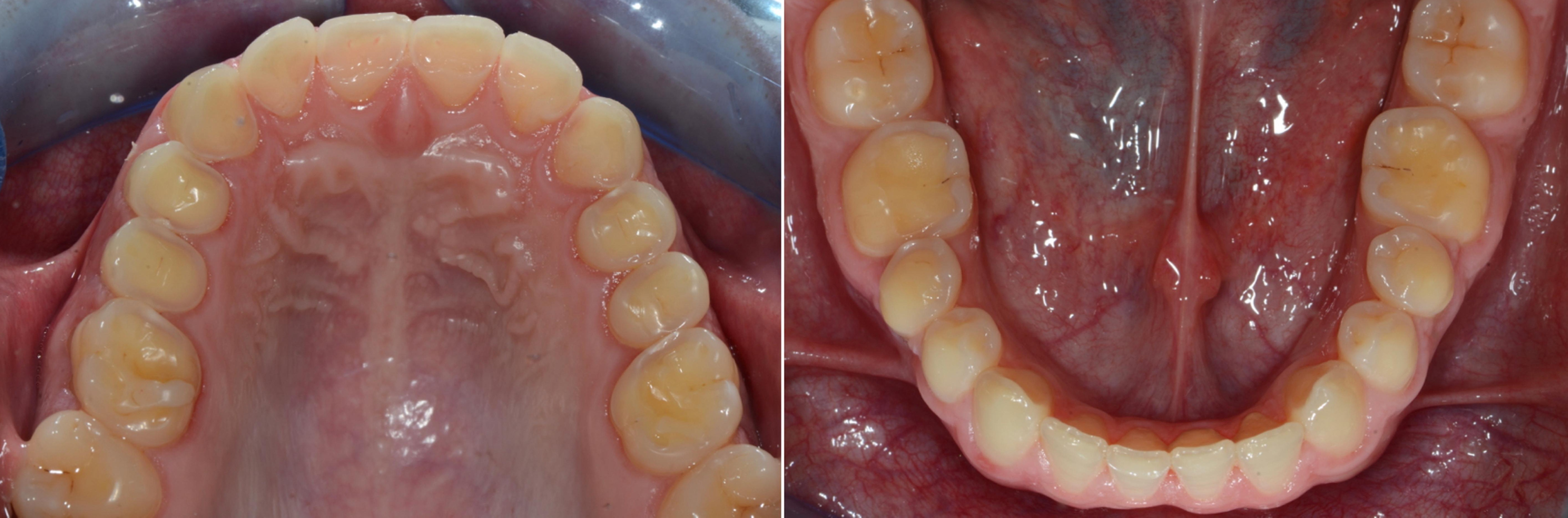




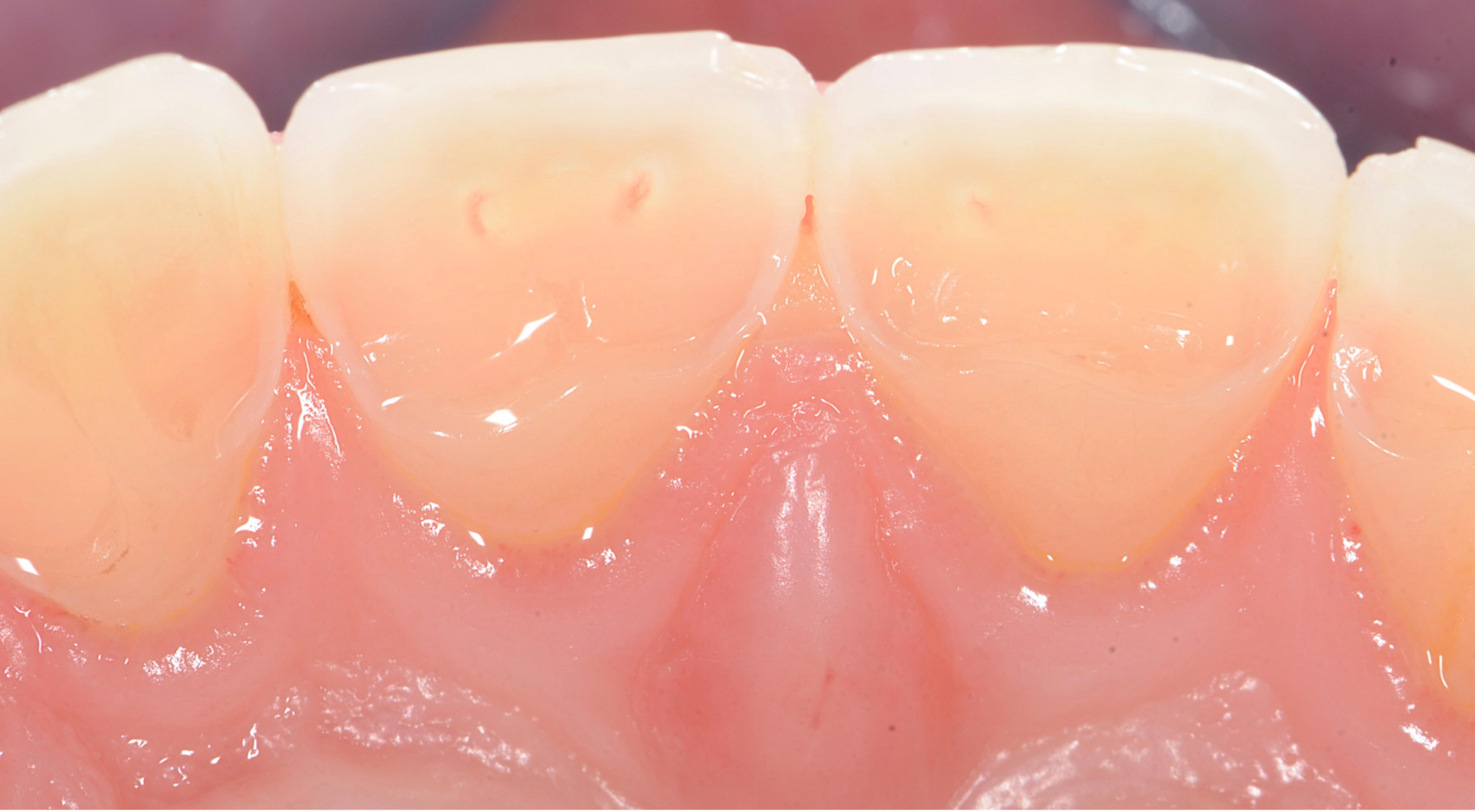


$1 \% \cdot 2 \sqrt{15}=$

- is is

\&.

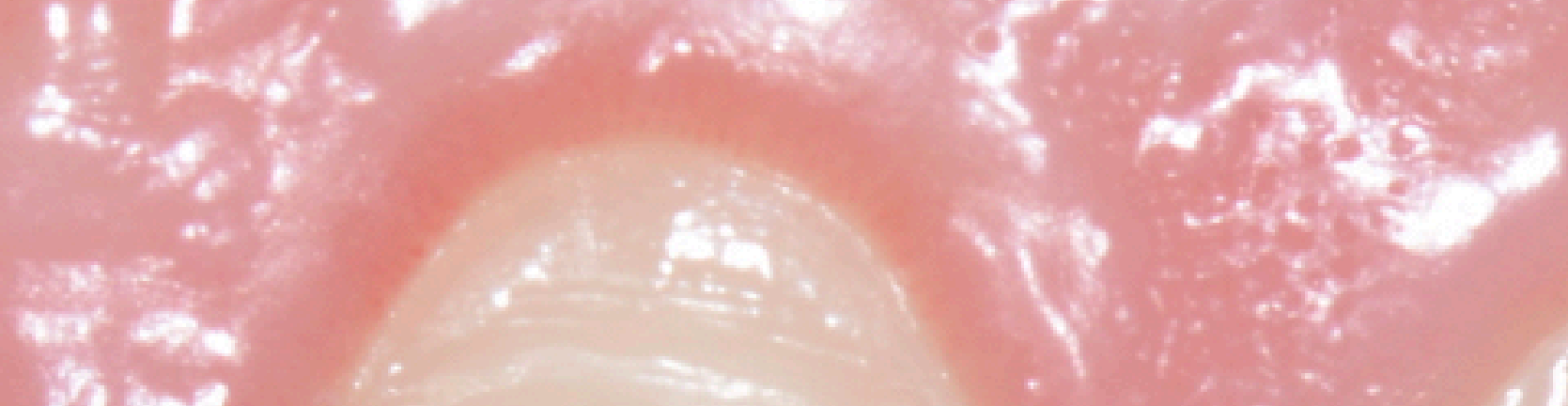

int

$+2$ (1) $\therefore$ तो

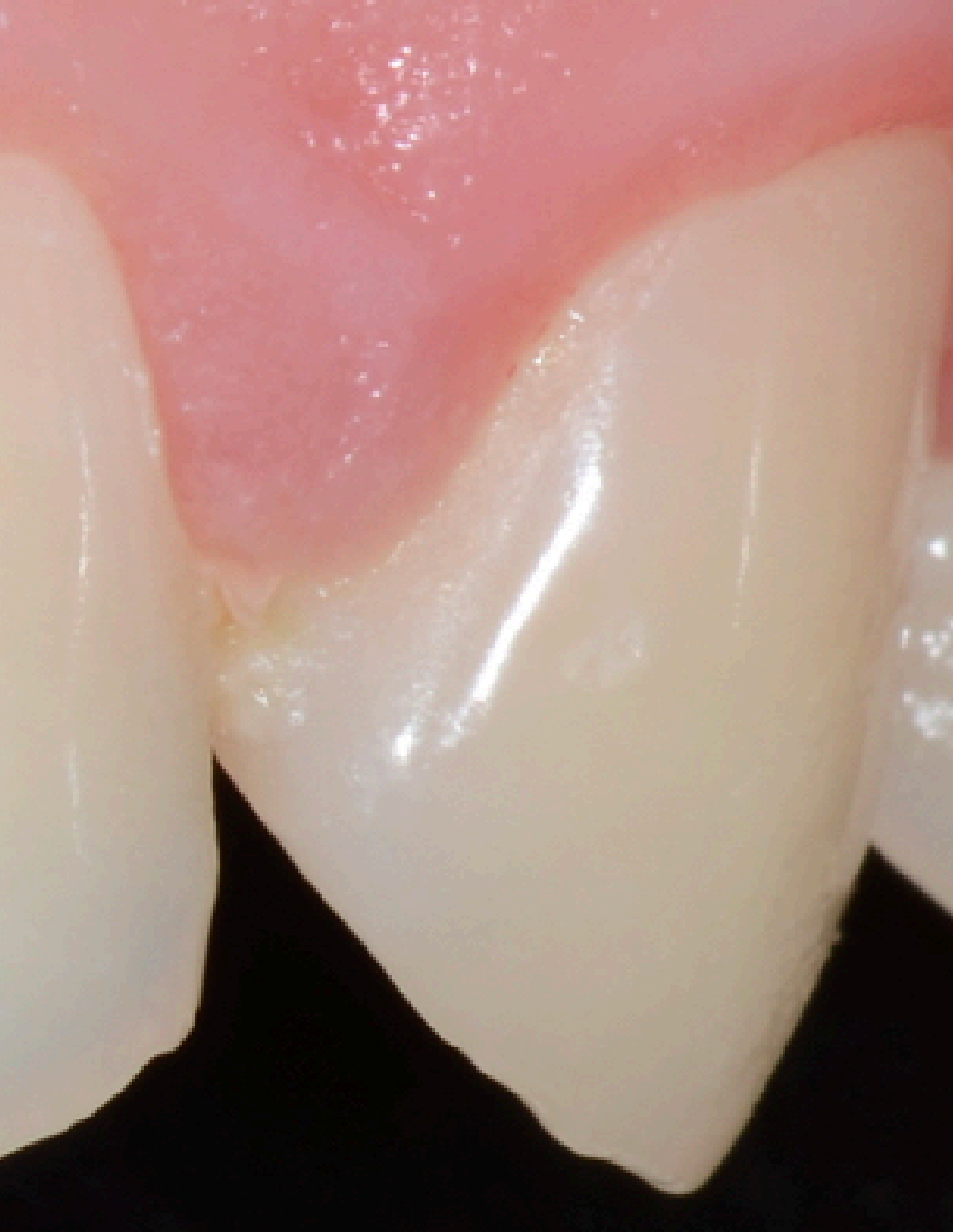


\&.

L. ? $80^{\circ}$

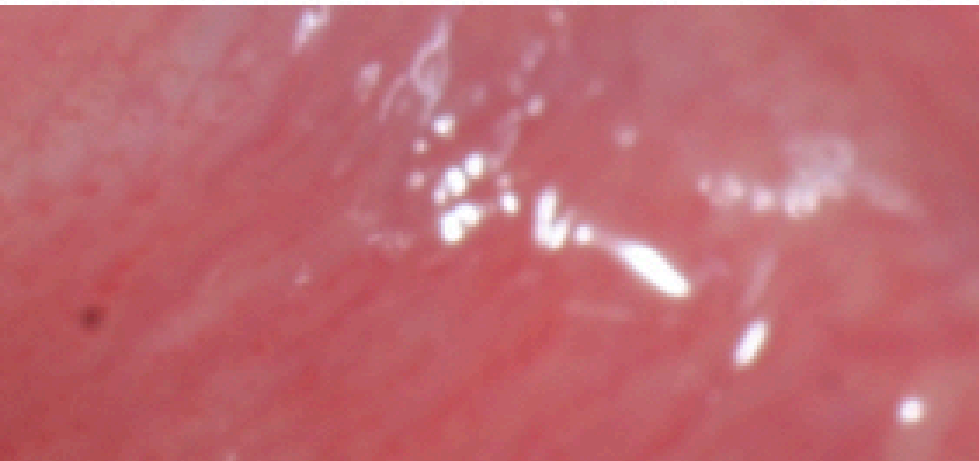

"1n

$\because 1$

1

.. $\frac{4}{4}$

..............

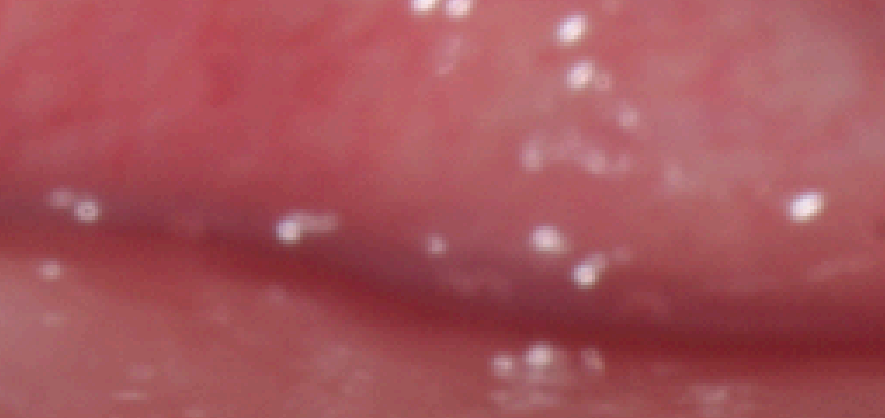

9

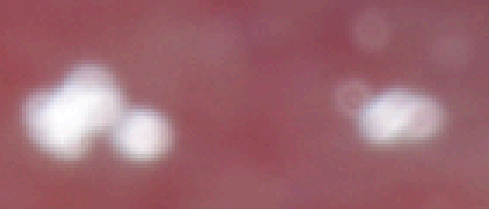

a

$-\infty$
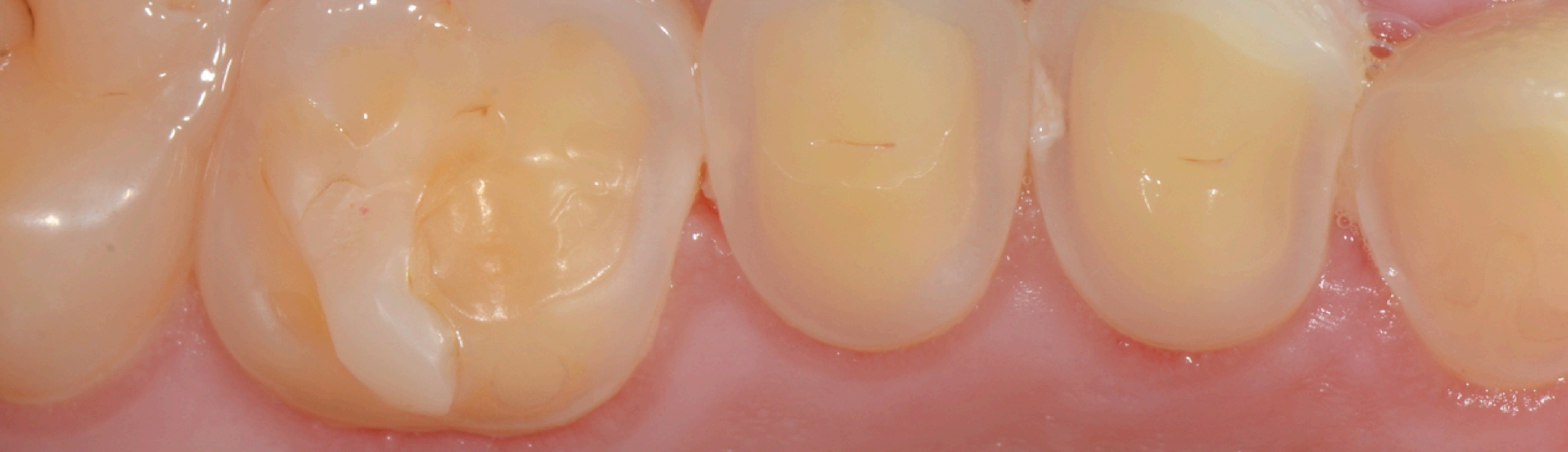


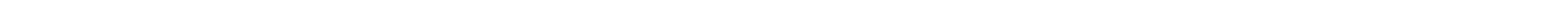




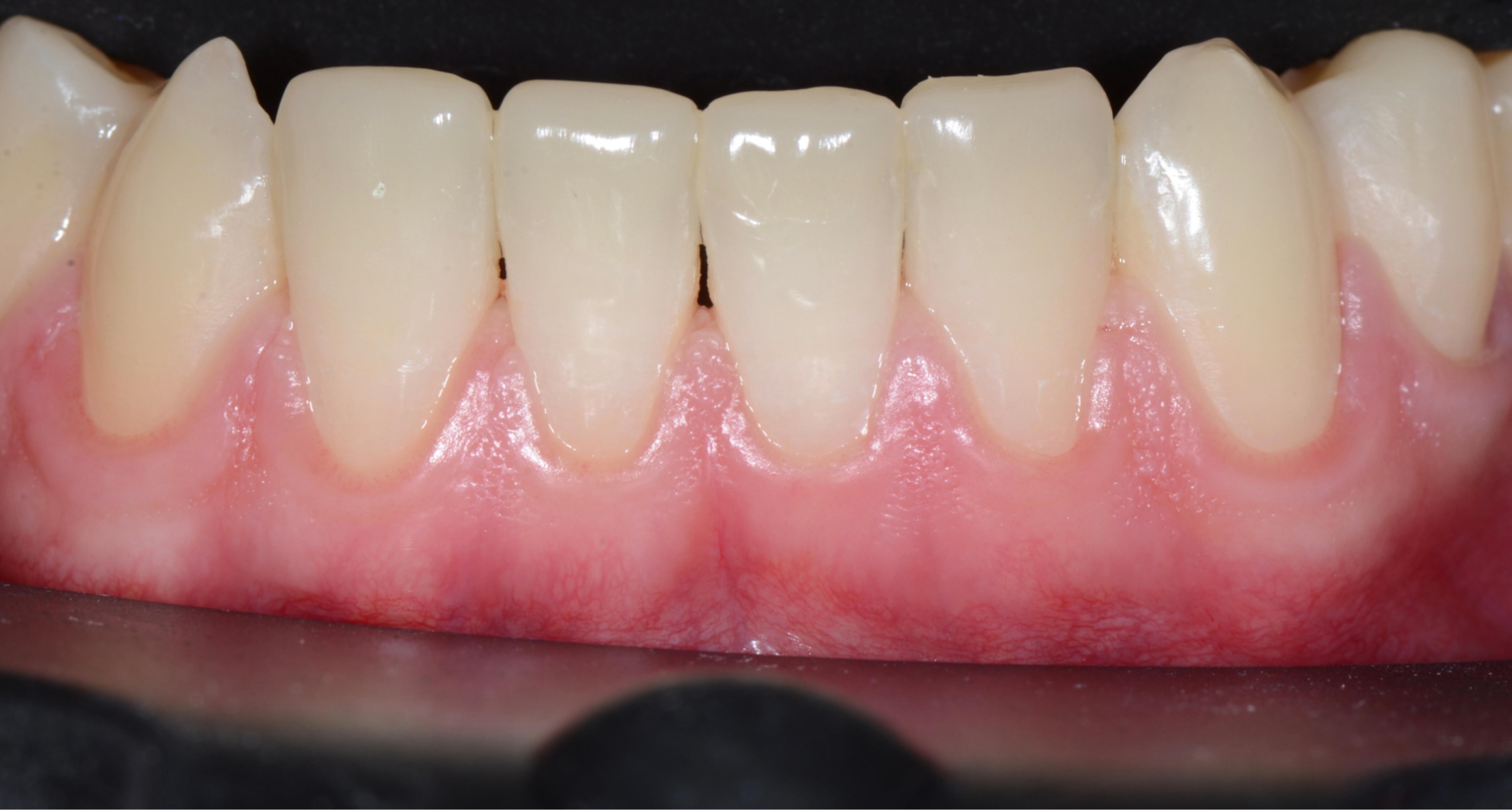




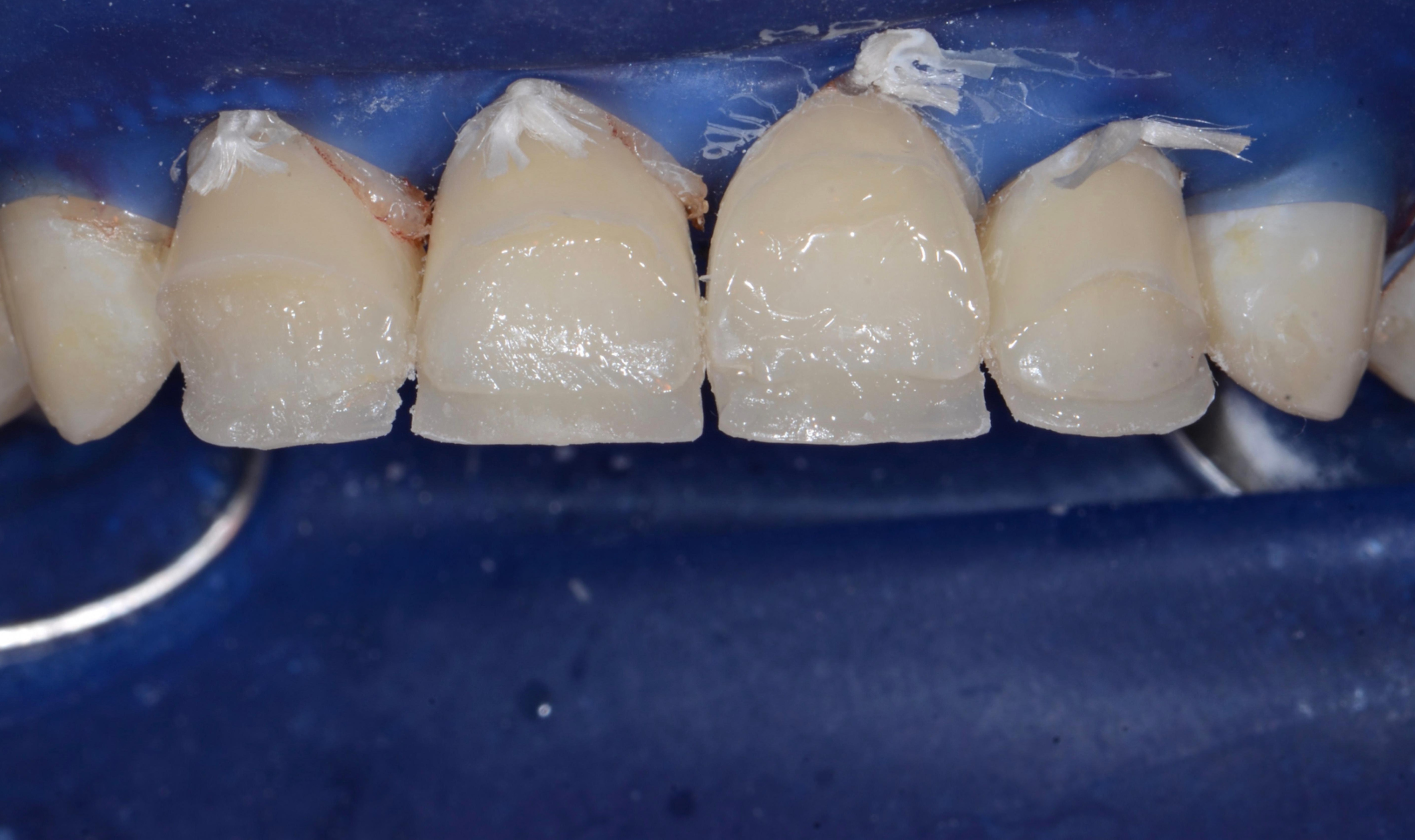




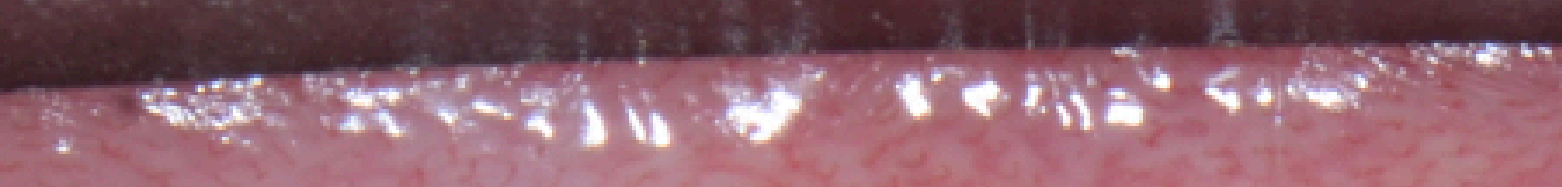




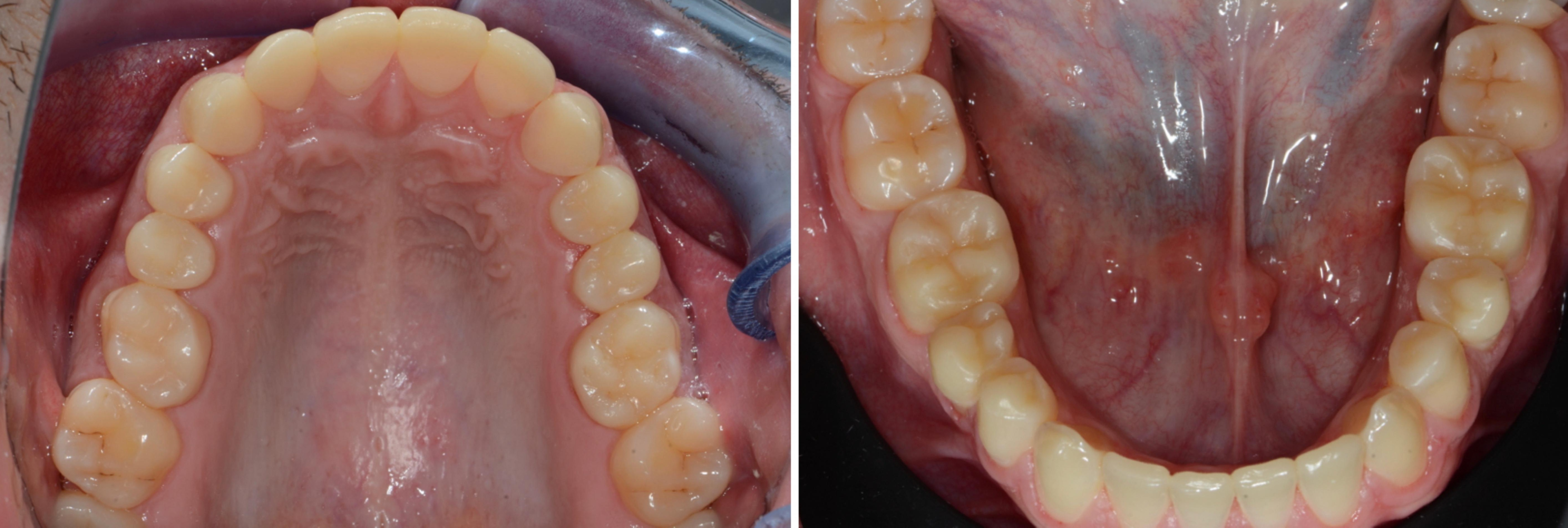

